\title{
Impact of Mediating Technologies on Talk and Emotion: Questioning "Commonsense"
}

\author{
Joon-Suk Lee \\ Virginia State University, Dept. of Computer Science \\ P.O. Box 9068, Petersburg, VA, USA \\ dolomite@vt.edu
}

\author{
Deborah Tatar \\ Virginia Tech, Dept. of Computer Science \\ MC 0106, 2002 Kraft Dr., Blacksburg, VA, USA \\ tatar@vt.edu
}

\begin{abstract}
Much CSCW research predominantly focuses on investigating how distributed, mediated interactions are different from collocated interactions, but rarely looks at how the use of technologies affect collocated people. We argue that the needs for studying the impact of mediating technologies among collocated people are current and large. A situation of seeing the other but not being able to see what captures his/her attention is endemic. In this paper, we investigate collocated triads as they play a collaborative, problem-solving game on laptops, on tablets or on a shared paper. People's positive emotion rose more when they talked about the complex relationships of the puzzle specifics and added new perspectives to other people's contributions during the game. People in computer conditions talked less about the specifics on the game board than people in the paper condition, but only people in the laptop condition experienced a significant decrease in positive emotion.
\end{abstract}

Keywords-component; Coordination; Collaboration; Form Factor; Mediating Technology; Group Interactions; Team Sudoku

\section{INTRODUCTION}

Increasing use of small screen technologies is creating opportunities to engage in highly coordinative activities for multiple users with multiple screens in collocated settings. A situation of seeing the other but not being able to see what captures his/her attention is endemic. Indeed, such situations will only increase as wearable computing devices become more and more ubiquitous. With devices such as Google Glass, we cannot even guarantee that what the other person sees is what I see even among the people within the same physical space. It will become enormously difficult to tell if a person is looking at me or looking at the personalized display projected onto his/her glasses. This has the potential to create many unexpected interactional problems. The needs for studying the impact of mediating technologies among collocated people are current and large.

Even though researchers studying computer mediated collaboration and coordination investigate how mediating technologies affect group processes, many are concerned with studying how technology-mediated, distance interactions are similar or different from non-technology-mediated, face-to-face interactions. Not much research investigates how various mediating technologies impact collocated people differently. Questions such as "would you feel more positive if you played a crossword puzzle with your friends on an iPAD as compared to playing the same game as printed in a newspaper or with a laptop?" or "would you talk more if you played Settlers of Catan on a laptop rather than playing it on a tablet?" are seldom asked. Furthermore, when asked, such questions are oftentimes considered obvious.

For instance, when we reported our preliminary findings [23] that collocated triads who played Sudoku on paper talked more than collocated triads who played the same game on computers, one of the responses we got was that "it is a trivial result to find that people talked more in the paper condition than in the other two conditions." We were told that the participants in the paper condition must have needed to exchange information whereas such information would automatically be logged in the laptop/tablet conditions.

However, one can also easily make a counter argument by saying that sharing a big piece of paper will enable every player to have immediate access to the visual information on the board, hence the need for further communication is precluded.

Perhaps for some people, it may seem that collocated interaction is commonsensical; however, as we will try to show throughout this paper, technologies influence people's behaviors and feelings in subtle but complex ways. The question of how technologies impact collocated groups is not only an important question to ask, but also an interesting one in the collaboration/coordination research field.

For instance, much previous research comparing video mediated communications (VMC) with face-to-face interactions has asked how different mediating technologies impact group discourse (e.g., $[1,2,28,40])$. Yet, their findings are somewhat inconclusive. Some studies report that communication technologies often create difficulties in turntaking among the geographically distributed, technologymediated groups, resulting in a decrease in the number of turns and an increase in the length of turns compared to face-to-face interactions $[28,40]$. Such research typically reasons that the grounding process takes longer in computer-mediated settings and therefore people using computers end up talking more. However, some other research either finds no such result [1] or finds contradictory results [2].

As with these previous studies, we are also interested in the impact that mediating technologies have on people's verbal behaviors. In our study, however, we look at how mediating technologies influence collocated groups. In particular, we 
explore the relation between users' emotional states, talk, and differences in the mediating technology.

In this paper, we report on our investigation of triads playing a highly demanding Collaborative Sudoku game as in [24], but we contrast Paper, Tablet and Laptop conditions. Questions we address in this paper include the following:

(1) How does the difference in the technological medium influence collocated triads' contributions to group discourse?

(2) How does the difference in the technological medium influence collocated triads' mood changes?

\section{STUDIES ON GROUP WORK}

For years, researchers from different disciplines have studied how groups work together. Much research studied how different factors affect group performance. Social psychologists studied how individuals affect group process and outcome. Behavioral phenomena attributable to individuals such as social loafing [20], social traps [29] and process loss [38] have been identified as degraders of group performances. Personality characteristics such as dominance, anxiety, affiliation, and leadership are known to affect group interactions both negatively and positively $[35,36]$.

The relationship between group processes and tasks has also been extensively studied. Many of these findings indicate that the type of task plays an important role in determining the group actions [30,32]. For instance, groups become more active when discussing open-ended problems than when faced with problems that only have one correct answer [13]. Hackman studied the correlation between production/discussion/problem solving type tasks and the group outcome, and reported that the differences in task types account for up to $50 \%$ of the variance in group outcomes [17]. A similar correlation between the group interactions and reasoning/mechanical assembly/discussion type tasks has also been reported [8]. Group size [14] and the composition of the group [21] are also known to affect the group process and the outcome.

Studies also looked at the different interactional properties of group activities. By investigating girls playing hopscotch, Goodwin examined how people utilize gestures, eye-gaze and talk in carrying out joint activities [16]. Barron studied the relations between interactional/communicative properties and group performance by observing triads solving math problems [3]. Some also investigated how eye-gazing is used in signaling the end of conversational turns [4], and even in predicting turntaking [19].

Studies looked at how verbal communications affect group performances and group members' perceived satisfactions. While many previous studies were built on the premise that cooperative group work requires a certain minimal level of communication [22], a more recent study showed that nontalking is not diagnostic of dissatisfaction nor low performance [24]. In contrast, verbal equity among the group members has also been associated with higher performance [7].

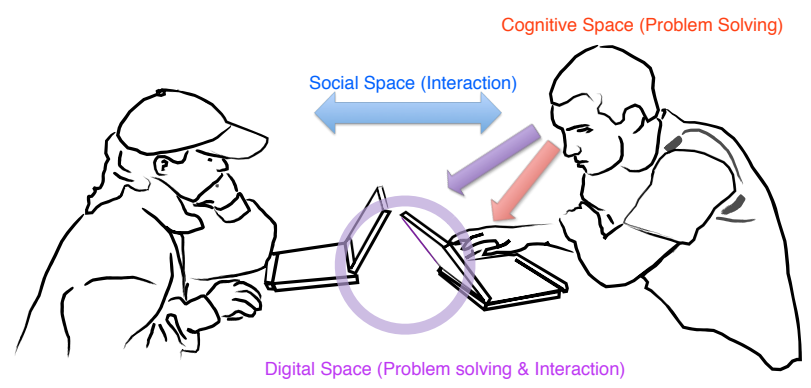

Figure 1: Attention and Task Management in Triple Space

Studies also explored how dyadic interactions are different from triadic interactions. For instance, [42] reported that triads outperformed dyads on a crypt-arithmetic problem solving task. Anderson and colleagues (c.f. [1,2]) examined how Clark's common ground model would apply to three-party interactions as compared to two-party interactions in both faceto-face and technology mediated settings, and reported not only that three-party interactions required more interactive work, but also that information distribution among the group members affected the grounding process.

While many have looked at the links between group performance and individual/organizational/interactional properties of groups and group members, not much research investigated the relationship between the choice of mediating technology and group processes. Although some researchers explored how mediated interactions among geographically distributed groups are different from face-to-face interactions $[1,2,28,40]$, not enough studies investigate how the differences in mediating technologies influence collocated people.

Previous research has shown that differences in interactive technologies can affect and change users' behaviors and their feelings toward the artifacts. For instance, people prefer a computer that flatters or diagnoses them as happy regardless of the correctness of the assessment [27]. People act politely towards computers in general [31], but not towards small computers [15]. However, these studies only look at interactions between individuals and computers, but not how individuals interact with one another through and around the technologies. In our study, unlike these studies that looked at how people react to different interactive technologies, we investigate how mediating technologies affect people in group settings.

In our study, we examine the role technological media play in influencing behaviors and emotional states of collocated triads in a "triple space" situation.

\section{TRIPLE SPACE INTERACTION}

The belief that people are experts in managing group activities with technology implies that the requirements are simple extrapolations of the already learned techniques. To the contrary, many tasks involve much more than simple extrapolations. For instance, Barron [3] describes how upper elementary school students solving math problems learn to manage a dual space: social and cognitive.

In a dual space, people need to manage their internal production of 'computational thinking,' and their social skills 
in managing group processes. Dual space tasks frequently involve asking a group to perform a task often thought of as belonging to the individual. Barron points out that joint problem-solving in dual space requires each participant to make his/her own thinking visible to the group, and to recognize other participant's thinking. She shows that not all groups know how to work together in dual space, and suggests that communication skills should be taught [3].

In our research, we look at an even more complex yet increasingly pervasive situation: a triple factor space (Fig. 1) in which people must manage not only the cognitive and social functions described by Barron, but also the challenge of making sense of the changing representations created by the other people on the shared medium.

This situation enacts a constellation of three increasingly important properties that often co-exist: (1) participant choice about the definition of "current purposes" inherent in the situation - that is, people may satisfice (in Simon's terms [34]) between goals or sacrifice one (they may not be optimizing); (2) participant choice about mechanisms of engagement with others, that is, people may choose where to direct attention and where not to direct attention; (3) tasks in which the effects of particular actions are not known or not known for a considerable lag. Often coordination research within HCI is concerned with whether one player can see what another player is seeing (as in [39]). The assumption is that seeing the action is sufficient to infer that action's meaning. But in these dual or triple factor spaces, there are two points of failure: the players may not perceive changes in state, or they may not understand the meaning of changes in state.

\section{THE STUDY}

To explore experimentally how differences in mediating technologies impact small groups' task performances, communicative behaviors and patterns as well as changes in the group members' individual emotional states, we asked groups of people to play Sudoku games collectively on one of three different mediating technologies. We asked players to play Sudoku puzzles on a 25 x 30.5 inch sheet (base-line, Paper Condition) and on two different form-configurations (Tablet Condition and Laptop Condition) of tablet PCs. In the paper condition (PC), researchers prepared the Sudoku game board manually prior to the study and asked participants to solve the puzzles on that. In both computer conditions, groups were asked to collaboratively solve puzzles on specially designed multiplayer Sudoku software, Team Sudoku.

We made an explicit decision to use a type of computer that has a twist-and-swivel display so that we could configure the same computer both as a laptop and as a tablet. For the laptop condition (LC), mice were connected to the systems as the primary input devices, while for the tablet condition (TC), stylus pens were provided as the primary input devices. Keyboard input mechanisms were disabled in the systems to maintain compatibility between the laptop condition and the tablet condition in which keyboards are hidden under the laiddown screens, inaccessible to the users.

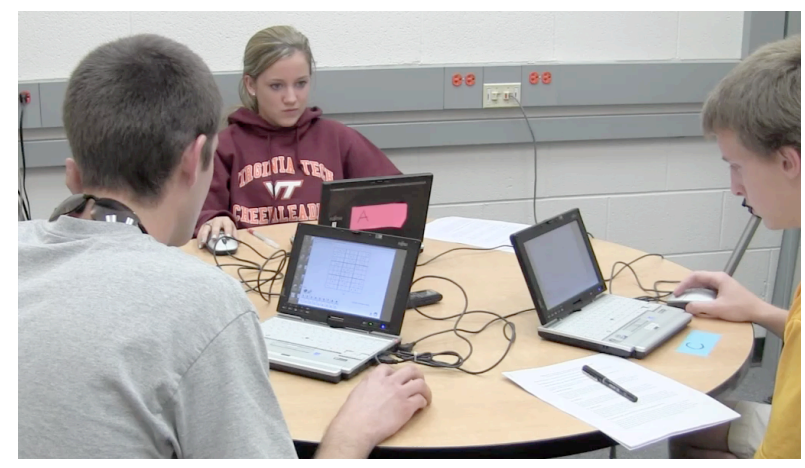

Figure 2: Set-up for three Team Sudoku Players (Laptop Condition shown).

Clearly, there are many differences between the paper and the computer conditions that are consequences of the types of sharing that they enable. However, the differences between LC and TC include only the form configuration and the input device.

\section{A. Team Sudoku}

Team Sudoku is a multi-user, parallel-distributed form of the Sudoku game. Sudoku presents the player with a 9x9 board with digits between 1 and 9 in some cells. The goal of Sudoku is to fill the board so that each of the nine columns, nine rows and nine distinct $3 \times 3$ blocks contain exactly one instance of each digit from 1 to 9 . Games are differentiated from one another by the number and location of starting digits. In the computerized form, each distinct game initially contains digits that cannot be written over or changed except by starting a new game, and that are a different color (black) from those that are in play (green).

Team Sudoku provides users three distinct features for manipulating the board. A pen tool enables users to insert entries on the board, and a pencil tool allows users to tentatively mark possibilities (note-entries). Users can delete any entries on the game board with an eraser tool. Each user can also highlight a row, a column, a $3 \times 3$ block, or a cell at a time using a personalized, color-coded highlighter tool.

Team Sudoku enables multiple players to interact with the game on their own computers, which are updated in real time. That is, when one player fills in a number, erases a number, or uses a highlighter, the results are immediately shared on all players' screens.

\section{B. Procedures}

The study was designed as a two-phased, between-subject experiment. During the initial sign-up process, participants were directed to an online survey site and asked to fill out questionnaires about demographics, prior experiences with Sudoku as well as five personality self-report inventories (Big Five [18], Circumplex Scales of Interpersonal Values [25], Circumplex Scales of Interpersonal Efficacy [26], Beck Anxiety Inventory [5] and Beck Depression Inventory [6]). Only the participants who filled out the online questionnaires were allowed to sign-up for phase 2. When participants reported that they did not have prior Sudoku experience, they 
were directed to a web site that had both descriptions of the Sudoku rules and sample Sudoku games.

In phase 2, participants were brought into a small room and seated in close proximity to one another (Fig. 2). They were introduced to one another when they came into the room. After the informed consent process, participants were asked to fill out a pre-game questionnaire (Q1) including questions about their experiences with the game and with the other players, and also the 20-item Positive and Negative Affect Schedule (PANAS) [41] that measures how people are feeling in the moment. After filling out Q1, researchers briefly went over the Sudoku rules (in all conditions) and conducted a software walkthrough (in LC and TC) in order to familiarize participants with the Team Sudoku tool features. The groups were then asked to work together on two Sudoku puzzles, in an order counter-balanced across groups. The groups were given 20 minutes to work on each game. After each game, participants filled out post-game questionnaires (Q2 and Q3), including retaking the PANAS. In Q3, we also asked them to rate how much they were satisfied with the group and the way it worked together on a scale of 1 (not at all) to 7 (very much). There was also a short discussion at the end of the study. Video and audio recordings of all the game sessions were collected as well as computer logs and screen shot movies of the games.

\section{Participants}

Players were recruited from the Psychology Participant pool at our university, and received extra credit for participation. A total of 138 (75 female, 63 male) college students enrolled in the study, in 24 groups of two and 30 groups of three. Participants' age ranged from 18 to $41(\mathrm{M}=19$ $\mathrm{SD}=2.28) .10$ of 138 reported that their first language was not English, but none appeared to have difficulty because of this. Almost all the participants had prior experience with Sudoku. 15 reported initially that they did not know the Sudoku rules, but researchers confirmed that these 15 people were at least fully familiar with the Sudoku rules when they came in for the on-site experiment. Overall, participants reported playing Sudoku quite often (mode $=6, \mathrm{M}=5.35, \mathrm{SD}=1.51$ ) on a scale of 1 (rarely) to 7 (several times a day). If only two participants appeared at the agreed time, the game was run with them, resulting in 24 groups of two. In this paper, however, we only address the triads.

6 pairs out of 90 participants knew each other before coming to the study. There were no groups in which all three participants knew each other, and the six pairs were relatively well spread out among the three conditions: 1 in PC, 3 in LC, and 2 in TC. We excluded these 6 groups from the analysis to ensure that there would exist no interfering effect between the acquaintance status and the groups' behaviors. The basis for the exclusion is explained in the next section.

\section{METHODS}

Four video cameras recorded each session. With one camera facing each participant and one capturing the entire group, we were able to ensure that each player's facial expressions as well as bodily gestures were captured in the video recordings. An additional audio recorder was also used to capture the participants' conversations.

The 24 20-minute video recordings of the first Sudoku game for triads were transcribed using a slightly modified version of Chafe's prosodic transcription system [9], which focuses on information flow. Transcripts were arranged into intonation and conversational turn units. Descriptions of nonverbal gestures as well as critical changes on the Sudoku board were also added to the transcripts. The final transcripts were reviewed for accuracy several times with the log files and screen-captured videos.

\section{A. Measuring Changes in Emotion}

The first mood state (PANAS) questionnaire was given before the first puzzle, the second between the first and the second puzzle, the third after the second puzzle. As usual, the sum of scores for the Positive Affect (PA) items and the sum of scores for the Negative Affect (NA) items were calculated for each PANAS test. Differences in scores $(\triangle \mathrm{PA}$ and $\triangle N A)$ were calculated to monitor the mood changes after playing each game, but we only focus on the first game in this investigation.

\section{B. Measuring Performance}

While Sudoku puzzles typically have only one correct solution, it is not easy to definitively assess an unfinished game. That is, Sudoku is an all or nothing game in that a group that has two incorrect entries after 10 moves is not necessarily performing any worse than a group that has four incorrect entries at the same stage.

In our case, only 2 out of 30 groups finished the puzzle (Game1) within the given time. Fully acknowledging the arbitrariness of the assessment metrics, for practical reasons, we used the number of correct/incorrect entries and the number of filled/left-empty cells to measure relative performances among the groups. The formula we used is as follows.

$$
\text { Score }=\alpha * X+\beta * Y+\gamma * Z
$$

$\mathrm{X}$ denotes the number of correct entries, $\mathrm{Y}$ the number of incorrect ones, and $\mathrm{Z}$ the number of left-empty cells. $\alpha, \beta, \gamma$ are weight variables, and we used values, 30, -20, and -10 respectively. The formula penalizes both empty and incorrect entries, but does so more heavily for the incorrect entries, while rewarding the correct ones. The scores for the groups ranged from -560 to $1620(\mathrm{M}=366.25$ and $\mathrm{SD}=615.76)$.

\section{Measuring Amount of Talk}

The counts for the conversational turns as well as the utterances were extracted and computed from the transcripts. The number of conversational turns each player took during the game ranged from 11 turns to 247 turns $(\mathrm{M}=78.58, \mathrm{SD}=$ 55.38). The number of utterances ranged from 41 to $1676(\mathrm{M}=$ 522.97, $\mathrm{SD}=378.66$ ).

\section{Measuring Kinds of Talk}

Transcripts from 24 triad sessions were coded using the 3layered hierarchical coding scheme developed by Lee, Tatar 
and Harrison [24]. The coding scheme consists of 20 codes under four main categories and ten subcategories. The four main conversational categories are 'board-related,' 'gamerelated,' 'off-topic,' and 'other.'

Board-related utterances refer to specific elements, regions, possibilities, or problems on the Sudoku board. It has four subcategories. Statement represents simple, unelaborated statements or fragmented ideas such as "uh oh the six has to go there." Question refers to incidents in which a participant expresses doubts, asks for confirmation, or demands an explanation of elements on the game board such as "why does it have to be on the bottom?" Elaboration denotes cases in which a participant goes a little further to explain reasons for moves, possibilities and problems rather than just stating simple facts. Adding dimensionality is a code that captures instances in which participants build ideas on top of previously presented ones. In coding adding dimensionality, we added one more constraint to Lee, Tatar and Harrison's original adding dimensionality category [24] in order to prevent one player dominantly adding new ideas one after another from causing an over-estimated number for the adding dimensionality category. So in our case, for utterances to be considered as adding dimensionality, (1) segments of utterances needed to add new ideas to the group conversation, and (2) the person contributing the new ideas should not have contributed the immediately preceding new ideas. If a participant contributed two new ideas in a row, we considered the person to be leading the flow of the conversation, and marked the second new idea as board-related statement, question or elaboration based on the nature of the utterance.

Adam: I'll put like little fives here \{statement\}

Caitlin: but you still need

like a five in that row though

and it can't go anywhere else

in that [row] \{adding dimensionality\}

Adam: [why] do you need a five in that row \{question\}

Caitlin: ((begins to explain the reasons)) \{statement\}

Excerpt 1: a coding example - statement, question and adding dimensionality

In Excerpt 1, for instance, we code Adam's first turn as statement because Adam, by saying he will make changes to the game board, starts a new segment of discourse isolated from previous conversation. Caitlin adds a new perspective to Adam's proposed move by proposing other possibilities. Therefore, we code Caitlin's first turn as adding dimensionality. In his second conversational turn, Adam asks Caitlin why she said what she said, but adds no new perspective to the specifics of the game board. We code Adam's second turn as question. In her second turn, Caitlin adds previously unmentioned ideas to the group conversation. However, this time, we consider Caitlin to be taking over the flow of the conversation since she adds two consecutive new ideas, and code the turn as statement.

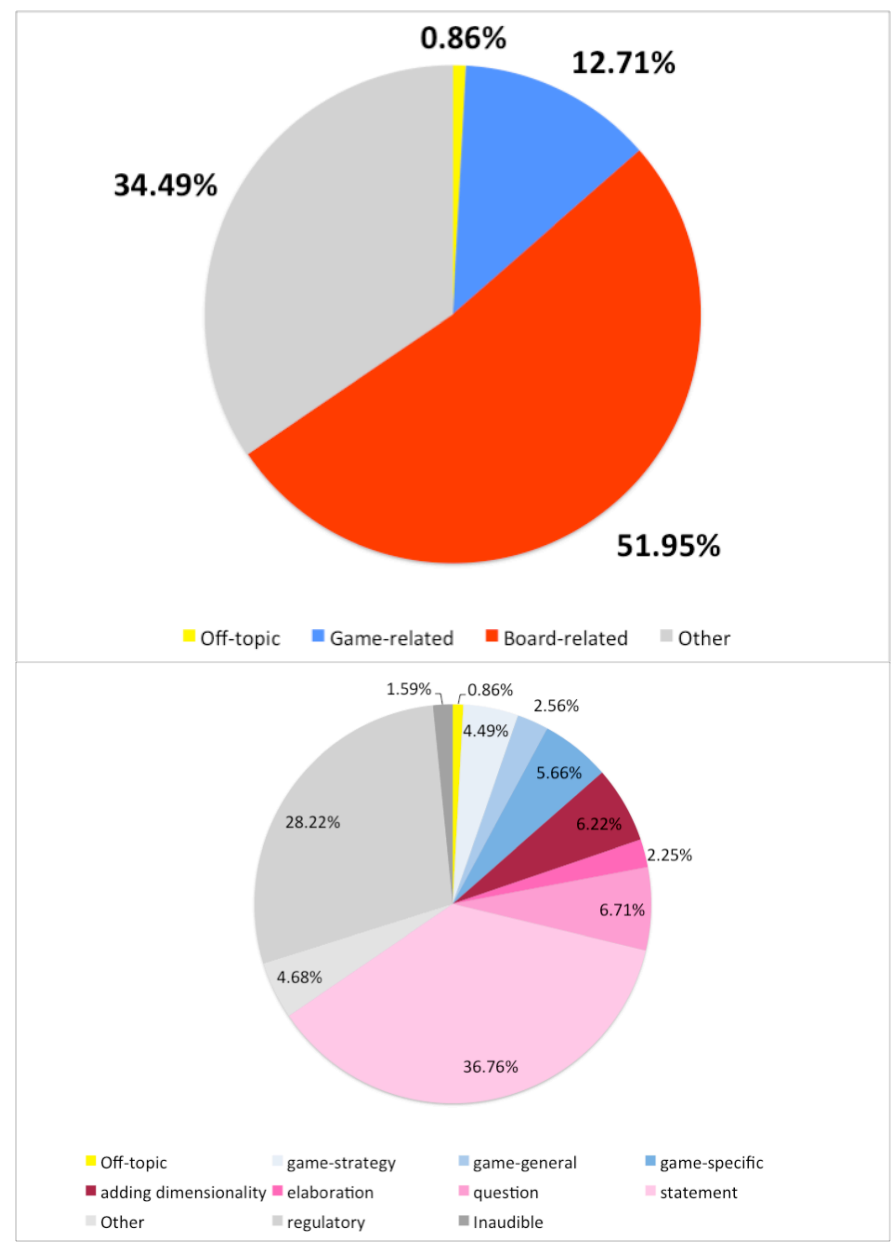

Figure 3. Overall Number of Utterances by Category.

Game-related utterances are closely related to the game, but do not refer to any specifics of the game board. This category includes discussion of game strategy, game-specific and gamegeneral discourses. Game-specific is discourse related to the current puzzle but not to specifics of the puzzle (as in "this puzzle is hard"). Game-general utterances denote inquiries and comments on Sudoku rules, prior Sudoku experiences, software features and research procedures.

Off-topic included utterances not directly related to the game activities. Inaudible, regulatory intonations (non-lexical and phrasal backchannels), and non-sentential sounds (e.g., laughs) were coded as Other.

Fig. 3 shows the distribution of utterances in the top two levels of the coding scheme. Only $0.86 \%$ of the conversation was off-topic, suggesting that people were quite engaged in the puzzle solving activities. $28.22 \%$ of the utterances were back channels. $36.76 \%$ were simple board-related statements. 


\section{FINDINGS}

\section{A. Triads with only strangers vs. Triads that had acquainted pairs}

As mentioned previously, 6 pairs out of 90 participants knew each other before coming to the study. Even though these 6 pairs were well spread out among the three conditions, we were still curious if the groups with only strangers were any different from the groups that had acquainted pairs.

Even though a Chi-square test for independence indicated no significant association between the condition and acquaintance status $\left(\chi^{2}(2, \mathrm{n}=90)=3.75, \mathrm{p}=.15\right.$, Cramer's V $=.2$ ), an independent-samples t-test showed a statistically significant difference between the two types of groups. When we compared the changes in participants' positive emotions for groups that had pairs who knew each other $(\mathrm{M}=4.11, \mathrm{SD}=$ $6.77)$ and groups that $\operatorname{did} \operatorname{not}(\mathrm{M}=0.68, \mathrm{SD}=6.09)$, these two types of groups were significantly different $(\mathrm{t}(88)=2.09, \mathrm{p}=$ .04 , two-tailed).

In other words, after playing the first Sudoku game together, the groups with acquainted pairs felt significantly happier than the groups with only strangers. Hence we dropped the six groups from the subsequent analysis.

\section{B. How the difference in the technological medium}

influences participants' contributions to group discourse

We previously reported that people talked more when they shared a big piece of paper to collectively work on a Sudoku puzzle compared to when they were using computers [23].

Our new analyses showed that statistical differences existed not only in the overall amount of talk, but also in the distribution of kinds of utterances. The amount of boardrelated conversation $(\mathrm{F}(2,21)=3.40 ; \mathrm{p}=0.05)$ differed by condition. Post-hoc comparisons showed that groups in PC (paper condition) talked significantly more about the specifics of the game board than groups in TC (tablet condition) (PC vs. TC: Mean Dif. $=115.08, p<0.05)$ and marginally more than groups in LC (laptop condition) (PC vs. LC: Mean Dif. = 87.33, $\mathrm{p}=0.08)$.

It is, perhaps, not very surprising that participants in PC had more board-related conversation than participants in the other two conditions considering our previous finding that people in PC talked more overall than people in the other two conditions. The more the participants talked, the more likely they were to talk certain kinds of conversation. However, more interesting differences lay in the proportional comparisons for the different kinds of talk. When we looked at the percentage of different discourse categories, participants in $\mathrm{PC}$ had proportionally more conversation marked as board-related $(\mathrm{F}(2,21)=5.81, \mathrm{p}$ $<0.01)$ than people in the other conditions. In addition, somewhat analogous to Anderson's findings that collocated, non-technology-mediated groups had significantly less strategy discussion than distributed, technology-mediated groups [1], people in PC had proportionally less conversation coded as game-strategy $(\mathrm{F}(2,21)=4.03, \mathrm{p}<0.05)$ than the other conditions. Table 1 summarizes the post-hoc analyses between the pairs.
TABLE I. POST-HOC ANALYSIS FOR KINDS OF TALK / TOTAL CONVERSATIONAL TURNS TAKEN BY GROUP

\begin{tabular}{|l|l|c|c|}
\hline Kinds of Conversation & Condition Pairs & Mean Diff. & p-value \\
\hline board-related (\%) & PC vs. TC & 0.20 & $<0.01$ \\
\hline board-related (\%) & PC vs. LC & 0.14 & $<0.05$ \\
\hline game-strategy (\%) & PC vs. TC & 0.04 & $<0.05$ \\
\hline game-strategy (\%) & PC vs. LC & 0.04 & $<0.05$ \\
\hline
\end{tabular}

In sum, participants in PC talked more about specifics of the game-board, but less about the game strategy than people in the other two conditions in both absolute and relative terms.

\section{How the difference in the technological medium influences participants' emotions}

Overall, a marginal positive correlation exists between the group average of the differences in members' positive emotional states $\left(\triangle \mathrm{PA}^{\text {group }}\right)$ and the average of members' contributions to discourse $(r=0.36 ; p=0.08)$. That is, groups that had participants who took more conversational turns overall tended to exhibit a higher rise in positive emotion. However, $\Delta \mathrm{PA}^{\text {group }}$ was not correlated with the number of contribution to the board.

More interesting correlations were between $\Delta \mathrm{PA}^{\text {group }}$ and different kinds of talk that groups held during the game. In general, a significant positive-correlation existed between $\triangle \mathrm{PA}^{\text {group }}$ and the number of conversational turns marked as adding dimensionality $(\mathrm{r}=0.41 ; \mathrm{p}<0.05)$ or as board-related $(\mathrm{r}=0.44 ; \mathrm{p}<0.05)$. There also existed a marginal negativecorrelation between $\triangle \mathrm{PA}^{\text {group }}$ and the number of conversational turns coded as game strategy $(\mathrm{r}=-0.36 ; \mathrm{p}=0.08)$. In other words, the more the groups talked about the complex relationships of the puzzle specifics and added new perspectives to other people's contributions, the higher the rise in the average of group members' positive emotions. The more they talked about game strategy, the less positive they felt. No correlation was found between the differences in people's negative emotional states $(\triangle \mathrm{NA})$ and any of the metrics we used in this investigation.

A one-way between-conditions analysis of variance was conducted to explore the impact of the technological conditions on the average of the changes in positive emotions $\left(\Delta \mathrm{PA}{ }^{\text {group }}\right)$. Since Levene's test for homogeneity of variances showed unequal variances among the three conditions $(p=0.15)$, we used Welch's Robust ANOVA. The result reported that differences existed in $\triangle \mathrm{PA}^{\text {group }}$ among the conditions ( $\mathrm{F}(2$, $13.53)=4.37, \mathrm{p}<0.05)$ in which PA scores showed a gain of the most positive affect in PC, a gain of only a bit in TC, and a loss in LC (Table 2).

Drilling down, post-hoc comparisons using the LSD test indicated that participants in $\mathrm{PC}$ had a significantly higher rise in PA scores than participants in LC (Mean Dif. $=4.29$; $\mathrm{p}<$ $0.05)$. Those who used paper were quite a bit happier than those who used the laptop. Subtle, but more surprising was the difference between TC and LC. The difference in $\triangle \mathrm{PA}^{\text {group }}$ between TC and LC was marginally significant (Mean Dif. = 2.64; $\mathrm{p}=0.15$ ). Instead of Laptop and Tablet resembling one 
another, Paper and Tablet were more aligned on this outcome measure. Furthermore, the mean value for $\mathrm{LC}(\mathrm{M}=-1.81)$ decreased after the game, while the mean value for TC $(\mathrm{M}=0.83)$ and $\mathrm{PC}$ increased $(\mathrm{M}=2.48)$.

In addition, individual level analyses also showed compatible results: overall differences existed in $\triangle \mathrm{PA}^{\text {individual }}$ among the conditions $(\mathrm{F}(2,69)=3.12, \mathrm{p}=0.05)$; participants in PC had a significantly higher rise in PA scores than participants in the LC (Mean Dif. $=4.29 ; \mathrm{p}<0.05$ ).

In sum, our data shows that the incremental changes in people's positive emotional states are associated with the properties of the medium. These differences are associated not only with the large and obvious differences between PC and the computer conditions, but also with the more subtle differences between LC and TC. The difference between LC and TC shows that not only the differences in mediating technology, but also the differences in form factor, affect people's emotional states differently.

\section{How the difference in the technological medium influences participants' self-reported satisfaction}

In investigating triads collaboratively playing Sudoku, Lee, Tatar and Harrison [24] showed that contributing to discourse was statistically related to participants' self-reported satisfaction. We were able to partially replicate their findings in this study.

In our case, the average number of conversational turns/group was 235.75, and the standard deviation was 140.38 . Overall, we observed a statistically significant difference in self-reported satisfaction related to the amount of discourse in the group. Players were asked to rate how satisfied they were with the group and the way they worked together on a scale of 1 (not at all) to 7 (very much). The number of conversational turns each player took was significantly correlated with selfreported satisfaction $(\mathrm{r}=0.281 ; \mathrm{p}=0.017)$. The more a player talked during the game, the higher satisfaction rate $\mathrm{s} / \mathrm{he}$ reported. However, there was no correlation between satisfaction rate and the number of entries individuals contributed to the game board $(\mathrm{r}=0.12 ; \mathrm{p}=0.45)$. Nor was satisfaction rate correlated with Sudoku scores $(\mathrm{r}=0.054 ; \mathrm{p}=$ $0.65)$.

TABLE II. Group Average of Positive AfFect Change Before to AFTER GAME1

\begin{tabular}{|l|c|c|c|}
\hline \multicolumn{1}{|c|}{ Condition } & $\boldsymbol{N}$ & Mean & Std.Err \\
\hline Paper (PC) & 9 & 2.48 & 1.56 \\
\hline Tablet (TC) & 8 & 0.83 & 0.98 \\
\hline Laptop (LC) & 7 & -1.81 & 0.69 \\
\hline
\end{tabular}

TABLE III. CROSS TABULATION ( $\triangle \mathrm{PA}^{\text {GROUP }} \mathrm{X}$ SCORE)

\begin{tabular}{|l|c|c|}
\hline \multicolumn{1}{|c|}{ Condition } & High Score & Low Score \\
\hline High $\Delta \mathrm{PA}^{\text {group }}$ & $\mathrm{G} 14(\mathrm{PC})$ & $\mathrm{G} 38(\mathrm{TC}), \mathrm{G} 40(\mathrm{PC})$ \\
\hline Low $\Delta \mathrm{PA}^{\text {group }}$ & None & $\mathrm{G} 34(\mathrm{TC}), \mathrm{G} 44(\mathrm{PC}), \mathrm{G} 46(\mathrm{LC})$ \\
\hline
\end{tabular}

When we ran the correlation tests by mediating technology conditions, the correlation between the self-reported satisfaction and the amount of talk was only evident in PC ( $\mathrm{r}=$ $0.448 ; p=0.019)$, but not in LC $(r=0.237 ; p=0.30)$, nor in TC $(\mathrm{r}=-0.146 ; \mathrm{p}=0.49)$. However, one-way ANOVA showed no significant differences among the three conditions $(F(2,69)$ $=1.04, p=0.36)$. This suggests that even though there exists correlation between self-reported satisfaction and the amount of conversational turns each individual took during the game session, no significant differences exist in the satisfaction rate among the three conditions. In other words, the differences in mediating technology seemed not to affect how individuals said they felt after playing Sudoku together.

\section{E. Interpreting Results}

From a statistical perspective, our data tells us that the differences in mediating technology affect participants' emotional states and verbal behaviors differently, but neither the game scores nor self-reported satisfaction is influenced by the conditions.

These statistical differences are, by themselves, important findings, but they do not lead to clean causal claims and associated design implications (even at the level of saying "use paper, and you will talk more, or use tablets instead of laptops, and you will be happier"). First, we do not know why people in PC would talk more about specifics on the game board. As we mentioned earlier, some might just say it is trivial that people in PC would just talk more since, both in LC and TC, all the game entry moves are automatically logged, and therefore people do not need to exchange information about the game entries. But is it really trivial?

Second, we cannot definitively tell why people who used tablets were less susceptible to the changes in positive emotions than people who used laptops. When participants used laptops, they talked relatively less about specifics on the game board, and had a considerable decrease in their positive feelings compared with people who used paper. But when participants used tablets, the changes in their positive feelings weren't statistically different from the people in the paper condition, even though people who used tablets also talked significantly less about specifics on the game board. We still do not know why people using tablets had higher positive emotions than people using the laptop. The fact that these two groups of people used exactly the same computers, but in different form configurations just hints to us that the form factor of technological medium might affect changes in people's emotional states differently.

To explore these issues, we conducted a contextualized analysis to develop fuller understandings of how the groups conducted their joint activities.

\section{HOW GROUPS DIFFER}

We first ranked groups based on Sudoku scores and $\Delta \mathrm{PA}^{\text {group }}$ into two ranking systems, and then grouped them into high (above the 3rd quartile), mid (below the 3rd and above the 1 st quartile), and low (below the 1st quartile). We then identified 6 sample groups based on the cross tabulation of the 
two ranking scales (Table 3). We base our qualitative discussions on these 6 groups.

The most salient difference between the high score, high $\Delta \mathrm{PA}^{\text {group }}$ group (group14) and three low score, low $\Delta \mathrm{PA}^{\text {group }}$ groups was how groups managed to maintain group focus.

Throughout the session, Ann, Bill and Chris (group14) maintained the group focus by utilizing not only verbal communication, but also physically putting their hands on the paper board. As shown in Fig. 4, these three players constantly pointed to same area on the puzzle board while speaking to each other. In this group, Ann worked as the dedicated entry marker for the team. She was the only one who marked down entries on the game board. Yet, the other two players also constantly pointed and kept their hands around the focused region of the game board.

Adam: like one of us could take the first three $=$ and then= just keep lookin and see if anybody filled in anything

Charles: well I usually just like $=$ do it like $=$.. all at once so like we can all just think together .. really

Adam: alright

Bobby: alright

Excerpt : Talking Strategy (Group44 - Paper Condition)

Group14 resembled a case of three boys that Brigid Barron described as "coordinated coconstruction" in her study [3] in that both groups maintained joint attention throughout the session and collaboratively worked toward solving the given tasks. However, the joint activity as well as the group discussions in group14 were led by two dominant players, Ann and Chris. In that sense, group14 also resembled "two's company" [3] since the group activity was led by two dominating participants. However, while the two dominating students in Barron's case ignored the less outspoken one, Ann and Chris, who contributed to $43 \%$ and $52 \%$ of the group discourse respectively, were always responsive to Bill's contributions. (Even though Bill only contributed to $5 \%$ of the group's discussion.)

On the contrary, group44 (also in PC) showed a distinctively different set of behaviors. Three male participants, Adam, Charles and Bobby, started their group activity by discussing how they would go about solving the puzzle. In Excerpt 2, Charles suggests they "do it like= all at once" (maintain group focus together) and "think together." However, what they did during the session was the exact opposite. Even though they sporadically focused on the same specifics on the game board and tried to talk through the solutions, they mostly remained silent, working individually. These three participants mostly kept their hands off the paper board. When they pointed to or wrote on the game board, they tended to work on separate regions as illustrated in Fig. 5. When multiple people needed to point to the same region, these three participants were hesitant to put their hands on the region already occupied by another player. Instead, they waited until the other player retracted his hands. This was quite distinctive from group14. In group14, both Ann and Chris continually approached the regions already occupied by other people's hands without any hesitation. Fig.
4., indeed, shows Ann placing her left hand onto the cell to which Chris is already pointing.

Groups in both the tablet and the laptop conditions obviously could not use their hands to point to the puzzle regions to indicate their focus. Even though we provided a deictic tool that could highlight different puzzle elements, people in both computer conditions used the tool much less frequently than people in the paper condition used their hands. Two notable shortcomings in the deictic tool were (1) it only supported highlighting one element at a time per player, while participants in PC had two hands per person, and (2) the deictic tool required activation (users needed to activate the highlighter to highlight and deactivate it to fall back into writing mode), while people's hands were readily available.

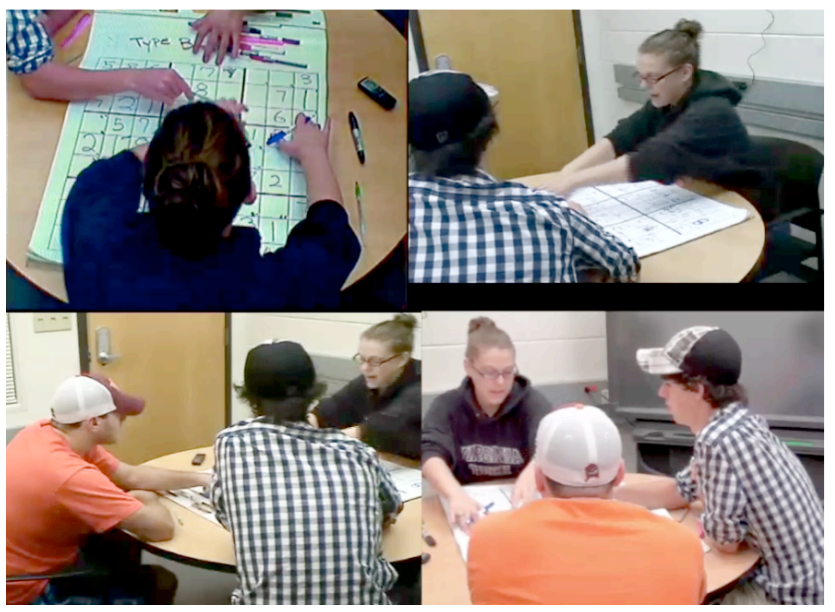

Figure 4: High $\triangle$ PAgroup, High Score - Group14 (Paper Condition)

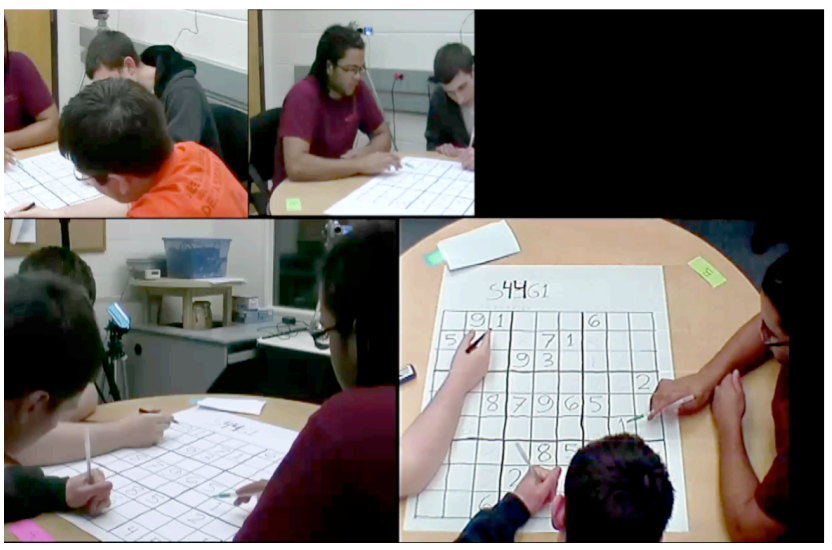

Figure 5: Low $\triangle$ PAgroup, Low Score - Group44 (Paper Condition)

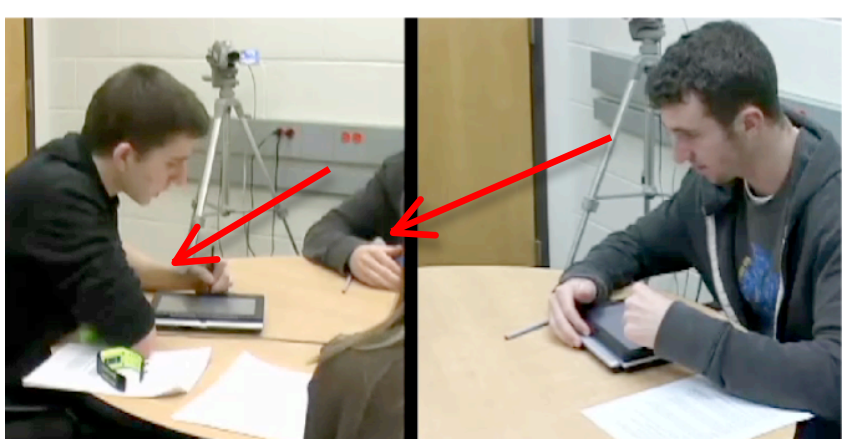

Figure 6: Glancing at Other Player's Screen (Tablet condition) 


John: go ahead and highlight it
Tom: how do you highlight?
do you [just-]
John: [uhm] you press the highlighter and then press that
Tom: alright

Excerpt 3: Discussion about a Tool Feature (Tablet Condition)

Different technology mediums afford different sets of behaviors and also have different constraints. Problems pertaining only to the paper condition exist. For example, since three people shared one big piece of paper, the game board was only oriented toward one person. The two other players had to look at the game board upside-down or side-ways. Participants handwrote the entries as well as small notes on the paper using pens. Some had difficulties reading their teammates' handwriting. However, even with all these shortcomings in the mediating technology, people in the paper condition experienced a rise in their positive emotional states. Sharing a physical game board might have helped people feel more connected than when they were sharing a digital game board. The digital mediums, by supporting surrogated interactions, might be pulling people away from more direct, social interactions. We cannot decisively tell what caused people in PC to feel significantly better than people in the other two conditions. Yet, it is most probable that different affordances in the mediating technology as well as in the deictic mechanism enabled different sets of behaviors, which impacted how people felt toward their group activities.

Although the statistical differences between TC and LC were not as prominently contrastive as the difference between $\mathrm{PC}$ and the computer conditions, there clearly existed subtle but important differences. Among the noticeable behavioral differences between TC and LC was that some participants in TC tried to glance at other players' screens during the sessions, while none in LC exhibited such behavior. For instance, in Excerpt 3, Tom asks John how to use the highlighter tool. As John explains how to enable the tool, Tom glances at John's screen very briefly (this moment is captured in Fig. 6). Even though players shared the same information on their own screens, participants might have felt more connected by having a chance to physically share what they considered to be their private resources. This conjecture might also help in explaining the highest mean numbers in positive affect changes in $\mathrm{PC}$, in which participants had a large shared resource. In LC, uprightpositioned screens might have acted as physical barriers between players, whereas in TC, laid-down screens could have helped people to feel enhanced social presence by providing increased immediacy for social interactions [35]. In fact, our data shows that participants in TC glanced at other people's screens significantly more than participants in LC (Mean Dif. = $2.74 ; \mathrm{p}<0.05)$.

\section{DISCUSSION AND CONCLUSION}

Our quantitative analysis shows statistically significant differences in the amount of particular kinds of talk among the three mediating technology conditions. When people shared a big piece of paper to collectively work on a Sudoku puzzle, they talked significantly more than people in the two computer conditions about the complex relationships of the puzzle specifics, adding new perspectives to other people's previous contributions.

Our data also show that people's positive feelings rose more when they talked more about the specifics of the game board. However, people who used tablets were less susceptible to the changes in positive emotions than people who used laptops. When participants used laptops, they talked less about the game contents than people who used paper, and their positive feelings decreased significantly. But when participants used tablets, the changes in their positive feelings weren't statistically different from the people in the paper condition, even though participants in the tablet condition also talked significantly less about the game contents than people in the paper condition.

Close examination of the group interactions revealed that participants in high performing groups in the paper condition utilized verbal communication as well as their hands to maintain group attention on specific regions of the game board throughout the session. Subtle behavioral differences also existed between the two computer conditions. Participants in the tablet condition sometimes glanced at other players' screens, while no such behavior was witnessed in the laptop condition. We conjecture that not only would having the shared visual space help collaborators understand the current state of their tasks better [17], but also having the mere possibility of sharing certain visual information would impact how people feel toward their group work.

However, there is no clear theoretical work that supports our conjecture. Social presence theory [33] says that different communication media afford interlocutors different degrees of awareness of the other party in communications, and argues that the face-to-face medium provides the most social presence, while mediated communication supports less. Others [37] associate the lack of social context cues in computer-mediated communications with the degraded communicative experiences. Similarly, media richness theory [11,12] defines face-to-face interactions as richest and computer-mediated communications as lean. Yet, these theories do not fully explicate how the use of mediating technologies in face-to-face settings would impact the interactants.

We believe that future research comparing technologies with different screen sizes will help us further test our hypothesis that having physically less obtrusive technologies can cause an increase in positive emotional states.

In this paper, we did not advance our findings into a general theory. Nonetheless, our findings show that what some might consider obvious is not at all trivial, and that the use of technology in triple space deserves much attention from the researchers in our field.

\section{ACKNOWLEDGEMENTS}

This work was supported by NSF Grant. IIS-1018607 HCCSmall. 


\section{REFERENCES}

[1] Anderson, A. H. (2006). Achieving understanding in face-to-face and video-mediated multiparty interactions. Discourse processes, 41(3), 251287.

[2] Anderson, A. H., Mullin, J., Katsavaras, E., Brundell, P., McEwan, R., Grattan, E., \& O’Malley, C. (1999). Multimediating multiparty interactions. Human Computer Interaction (INTERACT99), 313-320.

[3] Barron, B. (2003). When smart groups fail. The journal of the learning sciences, 12(3), 307-359.

[4] Bavelas, J. B. (2005). Appreciating face-to-face dialogue. AVSP2005, 1 .

[5] Beck, A.T. \& Steer, R. A. (1997). Beck Anxiety Inventory.

[6] Beck, A. T., Ward, C. H., Mendelson, M., Mock, J., \& Erbaugh, J. (1961). An inventory for measuring depression. Archives of general psychiatry, 4(6), 561.

[7] Borge, M., Ganoe, C. H., Shih, S. I., \& Carroll, J. M. (2012, February). Patterns of team processes and breakdowns in information analysis tasks. InProceedings of the ACM 2012 conference on Computer Supported Cooperative Work (pp. 1105-1114). ACM.

[8] Carter, L., Haythorn, W., Meirowitz, B., \& Lanzetta, J. (1951). The relation of categorizations and ratings in the observation of group behavior. Human Relations.

[9] Chafe, W. (1993). Prosodic and functional units of language. Talking data: Transcription and coding in discourse research, 33-43.

[10] Cohen, J. (1988). Statistical power analysis for the behavioral sciences. Routledge Academic.

[11] Daft, R. L., \& Lengel, R. H. (1983). Information richness. A new approach to managerial behavior and organization design

[12] Daft, R. L., \& Lengel, R. H. (1986). Organizational information requirements, media richness and structural design. Management science, 32(5), 554-571.

[13] Gladstein, D. L. (1984). Groups in context: A model of task group effectiveness. Administrative science quarterly, 499-517.

[14] Frank, F., \& Anderson, L. R. (1971). Effects of task and group size upon group productivity and member satisfaction. Sociometry, 135-149.

[15] Goldstein, M., Alsiö, G., \& Werdenhoff, J. (2002). The media equation does not always apply: People are not polite towards small computers. Personal and Ubiquitous Computing, 6(2), 87-96.

[16] Goodwin, C. (2000). Action and embodiment within situated human interaction. Journal of pragmatics, 32(10), 1489-1522

[17] Hackman, J. R. (1968). Effects of task characteristics on group products. Journal of Experimental Social Psychology, 4(2), 162-187.

[18] John, O. P. (1990). The "Big Five" factor taxonomy: Dimensions of personality in the natural language and in questionnaires. In L. Pervin (Ed.), Handbook of personality: Theory and research (pp. 66-100). Guilford Press.

[19] Jokinen, K., Nishida, M., \& Yamamoto, S. (2010, February). On eyegaze and turn-taking. In Proceedings of the Workshop on eye gaze in intelligent human machine interaction,(Egihmi'10). ACM, New York, NY, USA (pp. 118-123).

[20] Karau, S. J., \& Williams, K. D. (1993). Social loafing: A meta-analytic review and theoretical integration. Journal of personality and social psychology, 65(4), 681.

[21] Laughlin, P. R., \& Johnson, H. H. (1966). Group and individual performance on a complementary task as a function of initial ability level. Journal of Experimental Social Psychology, 2(4), 407-414.
[22] Leavitt, H. J. (1951). Some effects of certain communication patterns on group performance. The Journal of Abnormal and Social Psychology, 46(1), 38.

[23] Lee, J. S., \& Tatar, D. (2013). Form factor matters. In Proceedings of the ACM 2013 conference on Computer Supported Cooperative Work

[24] Lee, J. S., Tatar, D., \& Harrison, S. (2012). Micro-coordination: because we did not already learn everything we need to know about working with others in kindergarten. In Proceedings of the ACM 2012 conference on Computer Supported Cooperative Work

[25] Locke, K. D. (2000). Circumplex scales of interpersonal values: Reliability, validity, and applicability to interpersonal problems and personality disorders. Journal of Personality Assessment, 75(2), 249267.

[26] Locke, K. D., \& Sadler, P. (2007). Self-efficacy, values, and complementarity in dyadic interactions: Integrating interpersonal and social-cognitive theory. Personality and Social Psychology Bulletin, 33(1), 94-109.

[27] Nass, C. I., \& Yen, C. (2010). The man who lied to his laptop: what machines teach us about human relationships. Current.

[28] O'Conaill, B., Whittaker, S., \& Wilbur, S. (1993). Conversations over video conferences: An evaluation of the spoken aspects of videomediated communication. Human-computer interaction, 8(4), 389-428.

[29] Platt, J. (1973). Social traps. American psychologist, 28(8), 641-651.

[30] Poole, M. S., Seibold, D. R., \& McPhee, R. D. (1985). Group decision making as a structurational process. Quarterly Journal of Speech, 71(1), 74-102.

[31] Reeves, B., \& Nass, C. (1996). How people treat computers, television, and new media like real people and places. Cambridge university press.

[32] Shaw, M. E. (1971). Group dynamics: The psychology of small group behavior.

[33] Short, J., Williams, E., \& Christie, B. (1976). The social psychology of telecommunications.

[34] Simon, H. A. (1996). The sciences of the artificial. MIT press.

[35] Smith, R. J., \& Cook, P. E. (1973). Leadership in dyadic groups as a function of dominance and incentives. Sociometry, 561-568.

[36] Sorenson, J. R. (1973). Group member traits, group process, and group performance. Human Relations, 26(5), 639-655.

[37] Stroebe, W., \& Diehl, M. (1994). Why groups are less effective than their members: on productivity losses in idea-generating groups. European review of social psychology, 5(1), 271-303.

[38] Sproull, L., \& Kiesler, S. (1986). Reducing social context cues: Electronic mail in organizational communication. Management science, 32(11), 1492-1512.

[39] Tatar, D. G., Foster, G., \& Bobrow, D. G. (1991). Design for conversation: lessons from Cognoter. International Journal of ManMachine Studies, 34(2), 185-209.

[40] van der Kleij, R., Schraagen, J. M., Werkhoven, P., \& De Dreu, C. K. (2009). How conversations change over time in face-to-face and videomediated communication. Small Group Research, 40(4), 355-381.

[41] Watson, D., Clark, L. A. \& Tellegen, A., (1988). Development and validation of brief measures of positive and negative affect: The PANAS scales. Journal of personality and social psychology, 54(6), 1063-1070.

[42] Wiley, J., \& Jensen, M. (2006, July). When three heads are better than two. In Proceedings of the 28th annual conference of the cognitive science society (pp.2375-2380). 